\title{
A Characterization of Semilinear Surjective Operators and Applications to Control Problems*
}

\author{
Edgar Iturriaga, Hugo Leiva \\ Grupo de Matemática Aplicada, Departamento de Matemáticas, Universidad de Los Andes, \\ Mérida, Venezuela \\ E-mail: iturri@ula.ve and hleiva@ula.ve \\ Received April 28, 2010; revised July 30, 2010; accepted August 3, 2010
}

\begin{abstract}
In this paper we characterize a broad class of semilinear surjective operators $G_{H}: V \rightarrow Z$ given by the following formula $G_{H} w=G w+H(w), w \in V$, where $V, Z$ are Hilbert spaces, $G \in L(V, Z)$ and $H: V \longrightarrow Z$ is a suitable nonlinear function. First, we give a necessary and sufficient condition for the linear operator $G$ to be surjective. Second, we prove the following statement: If $\operatorname{Rang}(G)=Z$ and $H$ is a Lipschitz function with a Lipschitz constant $h$ small enough, then $\operatorname{Rang}\left(G_{H}\right)=Z$ and for all $z \in Z$ the equation $G w+H(w)=z$ admits the following solution $w_{z}=G^{*}\left(G G^{*}\right)^{-1}\left(I+H \circ G^{*}\left(G G^{*}\right)^{-1}\right)^{-1} z$. We use these results to prove the exact controllability of the following semilinear evolution equation $z^{\prime}=A z+B u(t)+F(t, z, u(t))$, $Z \in Z, u \in U, t>0$, where $Z, U$ are Hilbert spaces, $A: D(A) \subset Z \longrightarrow Z$ is the infinitesimal generator of strongly continuous semigroup $\{T(t)\}_{t \geq 0}$ in $Z, B \in L(U, Z)$, the control function $u$ belong to $L^{2}(0, \tau ; U)$ and $F:[0, \tau] \times Z \times U \longrightarrow Z$ is a suitable function. As a particular case we consider the semilinear damped wave equation, the model of vibrating plate equation, the integrodifferential wave equation with Delay, etc.
\end{abstract}

Keywords: Semilinear Surjective Operators, Evolution Equations, Controllability, Damped Wave Equation

\section{Introduction}

In this paper we characterize a broad class of semilinear surjective operators

$G_{H}: V \longrightarrow Z$ given by the following formula

$$
G_{H} w=G w+H(w), w \in V,
$$

Where $Z, V$ are Hilbert spaces, $G: V \longrightarrow Z$ is a bounded linear operator (continuous and linear) and $H: V \longrightarrow Z$ is a suitable non linear function in general nonlinear. First, we give a necessary and sufficient condition for the linear operator $G$ to be surjective. Second, we prove the following statement: If $\operatorname{Rang}(G)=Z$ and $H$ is a Lipschitz function with a Lipschitz constant $h$ small enough, then $\operatorname{Rang}\left(G_{H}\right)=Z$ and for all $z \in Z$ the equation

$G w+H(w)=z$, admits the following solution

$$
w_{z}=G^{*}\left(G G^{*}\right)^{-1}\left(I+H \circ G^{*}\left(G G^{*}\right)^{-1}\right)^{-1} z .
$$

We apply our results to prove the exact controllability of the following semilinear evolution equation

\footnotetext{
*This work was supported by the CDCHTA-PROJECT: C-1667-0905-AA
}

$$
z^{\prime}=A z+B u(t)+F(t, z, u(t)), z \in Z, u \in U, t>0,
$$

where $Z$ and $U$ are Hilbert spaces, $A: D(A) \subset Z \longrightarrow Z$ is the infinitesimal generator of strongly continuous semigroup $\{T(t)\}_{t \geq 0}$ in $Z, B \in L(U, Z)$, the control function $u$ belong to $L^{2}(0, \tau ; U)$ and $F:[0, \tau] \times Z \times U \longrightarrow Z$ is a suitable function. We give a necessary and sufficient condition for the exact controllability of the linear system

$$
z^{\prime}=A z+B u(t), z \in Z, u \in U, t>0 .
$$

Under some conditions on $F$, we prove that the controllability of the linear system (1.3) is preserved by the semilinear system (1.2). In this case the control $u \in L^{2}(0, \tau ; U)$ steering an initial state $z_{0}$ to a final state $z_{1}$ at time $\tau>0$ (using the non linear system (1.2)) is given by the following formula:

$$
u(t)=B^{*} T^{*}(\tau-t) W^{-1}(I+K)^{-1}\left(z_{1}-T(\tau) z_{0}\right),
$$

where $K: Z \rightarrow Z$ is non linear operator given by:

$$
K \xi=\int_{0}^{\tau} T(\tau-s) F\left(s, z_{\xi}(s),(S \xi)(s)\right) d s,
$$

and $z_{\xi}(\cdot)$ is the solution of (1.2) corresponding to the 
control $u$ define by:

$$
u(t)=(S \xi)(t)=B^{*} T^{*}(\tau-t) W^{-1} \xi, \quad t \in[0, \tau] .
$$

As an application we consider some control systems governed by partial differential equations, integrodifferential equations and difference equations that can be studied using these results. Particularly, we work in details the following controlled damped wave equation

$$
\left\{\begin{array}{l}
w_{t t}+c w_{t}-d w_{x x}=u(t, x)+f\left(t, u(t, x), w, w_{t}\right) \\
0<x<1 \\
w(t, 0)=w(t, 1)=0 \\
t \in I R
\end{array}\right.
$$

where $d>0, c \geq 0$ the distributed control $u \in L^{2}\left(0, t_{1}\right.$; $\left.L^{2}(0,1)\right)$ and the nonlinear term $f(t, w, v, u)$ is a function $f:\left[0, t_{1}\right] \times I R^{3} \rightarrow I R$. A physical interpretation of the nonlinear term $f\left(t, u, w, w_{t}\right)$ could be as an eternal force like in the suspension bridge equation proposed by Lazer and McKenna (see [1]).

The novelty in this work lies in the following facts: First, the main results are obtained by standard and basic functional analysis such as Cauchy-Schwarz inequality, Hahn-Banach theorem, the open mapping theorem, etc. Second, the results are so general that can be apply to those control systems governed by evolutions equations like the one studied in [1-3] and [4]. Third, we find a formula for a control steering the system from the initial state $z_{0}$ to a final state $z_{1}$ on time $\tau>0$, for both the linear and the nonlinear systems, which is very important from engineering point of view. Also, we present here a variational approach to find solutions of the semilinear equation $G w+H(w)=z$ which is motivated by the one used to prove the interior controllability for some control system governed by PDE's, see [5]. Finally, these results can be used to motivate the study of semilinear range dense operator in order to characterize the approximate controllability of evolution equations.

\section{Surjective Linear Operator}

In this section we shall presents a characterization of surjective bounded linear operator. To this end, we denote by $L(V, Z)$ the space of linear and bounded operators mapping $V$ to $Z$ endow with the norm of the uniform convergence, and we will use the following lemma from [6] in Hilbert space:

Lemma 1. Let $G^{*} \in L(Z, V)$ be the adjoint operator of $G \in L(V, Z)$. Then the following statements holds:

1) $\operatorname{Rang}(G)=Z \Longleftrightarrow \exists \gamma>0$ such that

$$
G^{*} z_{V} \geq \gamma z_{Z}, z \in Z \text {. }
$$

2) $\overline{\operatorname{Rang}(G)}=Z \Longleftrightarrow \operatorname{Ker}\left(G^{*}\right)=\{0\}$.
In the same way as definition 4.1.3 from [7] we define the following concept:

Definition 1. The generalize controllability gramian of the operator $G$ is define by:

$$
W=G G^{*}: Z \rightarrow Z .
$$

Theorem 1. An operator $G \in L(V, Z)$ is surjective if, and only if, the operator $W=G G^{*}$ is invertible. Under this condition, for all $z \in Z$ the equation

$$
G w=z
$$

admits the following solution

$$
w_{z}=G^{*}\left(G G^{*}\right)^{-1} z=G^{*} W^{-1} z .
$$

Moreover, this solution has minimum norm. i.e.,

$$
w_{z}=\inf \{w: G w=z, w \in V\},
$$

and $w_{z}=w, G w=z \Longleftrightarrow w_{z}=w$.

Proof Suppose $G$ is surjective. Then, from the foregoing Lemma there exists $\gamma>0$ such that

$$
G^{*} z_{V}^{2} \geq \gamma^{2} z_{Z}^{2}, z \in Z \text {. }
$$

Therefore,

$$
<G G^{*} z, z>=<W z, z>\geq \gamma^{2} z_{Z}^{2} \quad z \in Z .
$$

This implies that $W$ is one to one. Now, we shall prove that $W$ is surjective. That is to say

$$
R(W)=\operatorname{Range}(W)=Z \text {. }
$$

For the purpose of contradiction, let us assume that $R(W)$ is strictly contained in $Z$. Using Cauchy Schwarz's inequality and (2.5) we get

$$
W z \geq \gamma^{2} z_{Z}, \quad z \in Z
$$

which implies that $R(W)$ is closed. Then, from Hahn Banach's Theorem there exists $z_{0} \in Z$ with $z_{0} \neq 0$ such that

$$
\left\langle W z, z_{0}\right\rangle=0, \forall z \in Z .
$$

In particular, putting $z=z_{0}$ we get from (2.5) that

$$
0=\left\langle W z_{0}, z_{0}\right\rangle \geq \gamma^{2} z_{0}{ }^{2} .
$$

Then $z_{0}=0$, which is a contradiction. Hence, $W$ is a bijection and from the open mapping Theorem $W^{-1}$ is a bounded linear operator.

Now, suppose $W$ is invertible. Then, given $z \in Z$ we shall prove the existence of $w \in V$ such that $G w=z$. This $w$ can be taking as follows

$$
w_{z}=G^{*} W^{-1} z \text {. }
$$

In fact,

$$
G w_{z}=G G^{*} W^{-1} z=G G^{*}\left(G G^{*}\right)^{-1} z=z .
$$

Now, we shall see that the solution 
$w_{z}=G^{*}\left(G G^{*}\right)^{-1} z=G^{*} W^{-1} z$ of the Equation (2.2) has minimum norm. In fact, let $w \in V$ such that $G w=z$ and consider

$$
\begin{aligned}
& w^{2}=w_{z}+\left(w-w_{z}\right)^{2}=w_{z}{ }^{2} \\
& +2 \operatorname{Re}\left\langle w_{z}, w-w_{z}\right\rangle+w-w_{z}{ }^{2} .
\end{aligned}
$$

On the other hand,

$$
\begin{aligned}
& \left\langle w_{z}, w-w_{z}\right\rangle=\left\langle G^{*} W^{-1} z, w-w_{z}\right\rangle= \\
& =\left\langle W^{-1} z, G w-G w_{z}\right\rangle=\left\langle W^{-1} z, z-z\right\rangle=0 .
\end{aligned}
$$

Hence, $w^{2}-w_{z}^{2}=w-w_{z}^{2} \geq 0$.

Therefore, $w_{z} \leq w$, and $w_{z}=w$ if $w_{z}=w$.

Corollary 1. If an operator $G \in L(V, Z)$ is surjective, then the operator

$S: Z \longrightarrow V$ defined by:

$$
S \xi=G^{*} W^{-1} \xi,
$$

is a right inverse of $G$.i.e., $G \circ S=I$.

Definition 2. Under the condition of the above theorem the operator

$$
S=G^{*} W^{-1}: Z \longrightarrow V,
$$

is called the generalize steering operator.

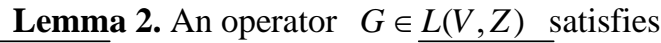

$\overline{\operatorname{Rang}(G)}=Z$ if, and only if, $\overline{\operatorname{Rang}(W)}=Z$.

Proof Suppose that $\overline{\operatorname{Rang}(G)}=Z$. Then, from Lemma 1 part (2) we have that

$$
\langle W z, z\rangle>0, \forall z \in Z, z \neq 0 .
$$

For the purpose of contradiction, let us assume that

$$
\overline{\operatorname{Rang}(W)} \subset Z \text {. }
$$

Then, from Hanh Banach's Theorem there exists $z_{0} \neq 0$ such that

$$
\left\langle W z, z_{0}\right\rangle=0, \forall z \in Z \text {. }
$$

In particular, if we put $z=z_{0}$, then $\left\langle W z_{0}, z_{0}\right\rangle=0$, which contradicts (2.7).

Now, suppose that $\overline{\operatorname{Rang}(W)}=Z$. Then, $\overline{\operatorname{Rang}\left(G G^{*}\right)}=Z$, and consequently $\overline{\operatorname{Rang}(G)}=Z$.

\subsection{Variational Method to Obtain Solutions}

The Theorem 1 gave a formula for one solution of the system (2.2) which has minimum norma. But, it is not the only way allowing to build solutions of this equation. Next, we shall present a variational method to obtain solutions of (2.2) as a minimum of the quadratic functional $\jmath: Z \rightarrow I R$,

$$
\jmath(\xi)=\frac{1}{2} G^{*} \xi^{2}-\langle z, \xi\rangle, \forall \xi \in Z .
$$

Proposition 1. For a given $z \in Z$ the Equation (2.2) has a solution $w \in V$ if, and only if,

$$
\left\langle w, G^{*} \xi\right\rangle-\langle z, \xi\rangle=0, \quad \forall \xi \in Z .
$$

It is easy to see that (2.9) is in fact an optimality condition for the critical points of the quadratic functional J define above.

Lemma 3. Suppose the quadratic functional $J$ has a minimizer $\xi_{z} \in Z$. Then,

$$
w_{z}=G^{*} \xi_{z},
$$

is a solution of (2.2).

Proof. First, observe that $\mathrm{J}$ has the following form

$$
\jmath(\xi)=\frac{1}{2}\left\langle G G^{*} \xi, \xi\right\rangle-\langle z, \xi\rangle, \quad \forall \xi \in Z .
$$

Then, if $\xi_{z}$ is a point where $J$ achieves its minimum value, we obtain that

$$
\frac{d}{d \xi}\{\jmath\}\left(\xi_{z}\right)=G G^{*} \xi_{z}-z=0 .
$$

So, $G G^{*} \xi_{z}=z$ and $w_{z}=G^{*} \xi_{z}$ is a solution of (2.2).

Remark 1. Under the condition of Theorem 1, the solution given by the formulas (2.10) and (2.3) coincide.

Theorem 2. The system (2.2) is solvable if, and only if, the quadratic functional $\jmath$ defined by (2.8) has a minimum for all $z \in Z$.

Proof Suppose (2.2) is solvable. Then, the operator $G$ is surjective. Hence, from Lemma 1 there exists $\gamma>0$ such that

$$
G^{*} \xi^{2} \geq \gamma^{2} \xi^{2}, \xi \in Z
$$

Then,

$$
\jmath(\xi) \geq \frac{\gamma^{2}}{2} \xi^{2}-z \xi, \xi \in Z .
$$

Therefore,

$$
\lim _{\xi \rightarrow \infty} \jmath(\xi)=\infty .
$$

Consequently, $\jmath$ is coercive and the existence of a minimum is ensured. The other way of the proof follows as in proposition 1.

\section{Surjective Semlinear Operators}

In this section we shall look for conditions under which the semilinear operator

$$
\begin{aligned}
G_{H}: V \longrightarrow & Z \text { given by: } \\
& G_{H} w=G w+H(w), \quad w \in V,
\end{aligned}
$$

is surjective. To this end, we shall use the following theorem from non linear analysis. 
Theorem 1. Let $Z$ be a Banach space and $K: Z \rightarrow Z$ a Lipschitz function with a Lipschitz constant $k<1$ and consider $\hat{G}(z)=z+K z$. Then $\hat{G}$ is an homemorphisme whose inverse is a Lipschitz function with a Lipschitz constant $(1-k)^{-1}$.

Theorem 2. If $\operatorname{Rang}(G)=Z$ and $H$ is a Lipschitz function with a Lipschitz constant $h$ such that $h G^{*}\left(G G^{*}\right)^{-1}<1$, then $\operatorname{Rang}\left(G_{H}\right)=Z$ and for all $z \in Z$ the equation

$$
G_{H} w=G w+H(w)=z,
$$

admits the following solution

$$
\begin{aligned}
& w_{z}=G^{*}\left(G G^{*}\right)^{-1}\left(I+H \circ G^{*}\left(G G^{*}\right)^{-1}\right)^{-1} z= \\
& =S(I+H \circ S)^{-1} z,
\end{aligned}
$$

where $S=G^{*}\left(G G^{*}\right)^{-1}$.

Proof Suppose that $\operatorname{Rang}(G)=Z$. Then, from Corollary 1 we know that the operator $S$ define by (2.6) is a right inverse of $G$, so if we put $G_{H}=G_{H} \circ S$, we get the new operator

$$
G_{H} \xi=G_{H} \circ S \xi=\xi+H(S \xi), \xi \in Z,
$$

where $S=G^{*}\left(G G^{*}\right)^{-1}=G^{*} W^{-1}$. Hence, if we define the operator $K: Z \longrightarrow Z$ by:

$$
K \xi=H(S \xi)
$$

the operator $G_{H}$ can be written as follows

$$
G_{H} \xi=\xi+K \xi=(I+K) \xi, \xi \in Z .
$$

On the other hand, $K$ is a Lipschitz function with a Lipschitz constant $\kappa \leq h G^{*}\left(G G^{*}\right)^{-1}<1$. Then, applying Theorem 1 we get the result.

Theorem 3. If $\operatorname{Rang}(G)=Z$ and the operator $K$ given by (3.5) is linear and $K \geq 0$, then $\operatorname{Rang}\left(G_{H}\right)=Z$ and for all $z \in Z$ the equation

$$
G_{H} w=G w+H(w)=z
$$

admits the following solution

$$
w_{z}=G^{*} W^{-1}(I+K)^{-1} z .
$$

Proof Since $G_{H} \circ S=I+K$, then

$$
\left\langle\left(G_{H} \circ S\right) z, z\right\rangle>z^{2}, \forall z \in Z .
$$

Then, in the same way as in the proof of Theorem 1 we get the result.

Corollary 1. Under the conditions of the above Theorems, the operator

$\Gamma: Z \longrightarrow V$ define by:

$$
\begin{aligned}
& \Gamma z=G^{*}\left(G G^{*}\right)^{-1}\left(I+H \circ G^{*}\left(G G^{*}\right)^{-1}\right)^{-1} z= \\
& =S(I+H \circ S)^{-1} z,
\end{aligned}
$$

is a right inverse of $G_{H}$. i.e., $G_{H} \circ \Gamma=I \mathrm{~d}$

Corollary 2. Under the conditions of the above
Theorems, the solution

$w_{z}=S(I+H \circ S)^{-1} z$ of the Equation (3.2) depends continuously on $\mathrm{Z}$. Moreover,

$$
w_{z}-w_{y V} \leq \frac{S}{1-h S} z-y_{Z}, \forall z, y \in Z .
$$

\section{Controllability of Semilinear Evolution Equations}

In this section we shall characterize the exact controllability of the semilinear evolution equation

$$
\begin{aligned}
& z^{\prime}=A z+B u(t)+F(t, z, u(t)) \\
& z \in Z, u \in U, t>0,
\end{aligned}
$$

Where $Z, U$ are Hilbert spaces, $A: D(A) \subset Z \longrightarrow Z$ is the infinitesimal generator of strongly continuous semigroup $\{T(t)\}_{t \geq 0}$ in $Z, B \in L(U, Z)$, the control function $u$ belongs to $L^{2}(0, \tau ; U)$ and $F:[0, \tau] \times Z \times U \longrightarrow Z$ is a suitable function.

\subsection{Linear Systems}

First, we shall study the controllability of the linear system (1.3), and to this end, for all $z_{0} \in Z$ and $u \in L^{2}(0, \tau ; U)$ the the initial value problem

$$
\left\{\begin{array}{l}
z^{\prime}=A z(t)+B u(t), t>0 \\
z(0)=z_{0},
\end{array}\right.
$$

admits only one mild solution given by:

$$
z(t)=T(t) z_{0}+\int_{0}^{t} T(t-s) B u(s) d s \quad t \in[0, \tau] .
$$

Definition 1. (Exact Controllability) The system (1.3) is is said to be exactly controllable on $[0, \tau]$, if for all $z_{0}, z_{1} \in Z$ there exists a control $u \in L^{2}(0, \tau ; U)$ such that the solution $z(t)$ of (4.3) corresponding to $u$, verifies: $z(\tau)=z_{1}$.

Consider the following bounded linear operator:

$$
G: L^{2}(0, \tau ; U) \rightarrow Z, G u=\int_{0}^{\tau} T(\tau-s) B u(s) d s,
$$

whose adjoint operator $G^{*}: Z \longrightarrow L^{2}(0, \tau ; U)$ is given by:

$$
\left(G^{*} \xi\right)(s)=B^{*} T^{*}(\tau-s) \xi, \forall s \in[0, \tau], \forall \xi \in Z .
$$

Then, the gramian $W=G G^{*}: Z \rightarrow Z$ takes the following classical form

$$
W z=\int_{0}^{\tau} T(\tau-s) B B^{*} T^{*}(\tau-s) z d s .
$$

Then, the following Theorem from [7](pg. 47, Theorem 4.17) is a characterization of the exact controllability of the linear system (1.3). 
Theorem 1. For the system (1.3) we have the following condition for exact controllability.

System (1.3) is exactly controllable on $[0, \tau]$ if, and only if, any one of the following condition hold for some $\gamma>0$ and all $z \in Z$ :

1) $G\left(L^{2}(0, \tau ; U)\right)=\operatorname{Range}(G)=Z$,

2) $\langle W z, z\rangle \geq \gamma\|z\|_{z}^{2}$,

3) $\left\|G^{*} z\right\|_{Z}{ }^{2}:=\int_{0}^{\tau}\left\|\left(G z^{*}\right)(s)\right\|_{U}{ }^{2} d s \geq \gamma\|z\|_{Z}{ }^{2}$

4) $\int_{0}^{\tau}\left\|B^{*} T^{*}(\tau-s) z\right\|_{U}^{2} \geq \gamma\|z\|_{z}^{2}$,

5) $\operatorname{Ker}\left(G^{*}\right)=\{0\}$ and $\operatorname{Rang}\left(G^{*}\right)$ is closed.

Remark 1. One can observe that the invertibility of the operator $W$ is not proved in the foregoing theorem and, consequently, none formula for the control steering the system (4.2) from initial state $z_{0}$ to a final state $z_{1}$ on time $\tau>0$ is given.

Now, we are ready to formulate and prove a new result on exact controllability of the linear system (1.3).

Theorem 2. The system (1.3) is exactly controllable on $[0, \tau]$ if, and only if, the operator $W$ is invertible. Moreover, the control $u \in L^{2}(0, \tau ; U)$ steering an initial state $z_{0}$ to a final state $z_{1}$ at time $\tau>0$ is given by the following formula:

$$
u(t)=B^{*} T^{*}(\tau-t) W^{-1}\left(z_{1}-T(\tau) z_{0}\right) .
$$

Proof It follows directly from the above notation and applying Theorem 1.

Corollary 1. If the system (1.3) is exactly controllable, then the operator

$$
\begin{aligned}
& S: Z \longrightarrow L^{2}(0, \tau ; U) \text { define by: } \\
& \quad S \xi=G^{*} W^{-1} \xi \text { or }(S \xi)(s)=B^{*} T^{*}(\tau-s) W^{-1} \xi
\end{aligned}
$$

is a right inverse of $G$. i.e., $G \circ S=I$

In this case the Equation (2.2) takes the following form

$$
\begin{aligned}
& G u=z \\
& u \in L^{2}(0, \tau ; U), z \in Z
\end{aligned}
$$

and the quadratic functional $\jmath$ given by (2.8) can be written as follows

$$
\mathfrak{I}(\xi)=\frac{1}{2} \int_{0}^{\tau} B^{*} T^{*}(\tau-s) \xi^{2} d s-\langle z, \xi\rangle, \xi \in Z .(
$$

The following results follow from Proposition 1, Lemma 3 and Theorem 1 respectively.

Proposition 1. For a given $z \in Z$ the Equation (4.9) has a solution $u \in L^{2}(0, \tau ; U)$ if, and only if,

$$
\int_{0}^{\tau}<u(t), B^{*} T^{*}(\tau-t) \xi>d s=<z, \xi>, \forall \xi \in Z .
$$

It is easy to see that (4.11) is in fact an optimality condition for the critical points of the quadratic functional $\mathfrak{I}$ define above.
Lemma 1. Suppose the quadratic functional $\mathfrak{I}$ has a minimizer $\xi_{z} \in Z$. Then,

$$
u(t)=B^{*} T^{*}(\tau-t) \xi_{z}, t \in[0, \tau],
$$

is a solution of (4.9).

Theorem 3. The system (1.3) is exactly controllable if, and only if, the quadratic functional $\mathfrak{I}$ define by (1) has a minimizer $\xi_{z}$ for all $z \in Z$.

Moreover, under this condition we obtain that

$$
\begin{aligned}
u(t) & =B^{*} T^{*}(\tau-t) \xi_{z}=B^{*} T^{*}(\tau-t) W^{-1} z, t \in[0, \tau], \\
\text { and } \xi_{z} & =W^{-1} z
\end{aligned}
$$

\subsection{Nonlinear System}

We assume that $F$ is good enough such that the Equation (4.1) with the initial condition $z(0)=z_{0}$ and a control $u \in L^{2}(0, \tau, U)$ admits only one mild solution given by:

$$
\begin{aligned}
& z(t)=T(t) z_{0}+\int_{0}^{t} T(t-s) B u(s) d s+ \\
& \int_{0}^{t} T(t-s) F(s, z(s), u(s)) d s, t \in[0, \tau]
\end{aligned}
$$

Definition 2. (Exact Controllability) The system (4.1) is said to be exactly controllable on $[0, \tau]$, if for all $z_{0}, z_{1} \in Z$, there exists a control $u \in L^{2}(0, \tau ; U)$ such that the corresponding solution $Z$ of (4.14) satisfies $z(\tau)=z_{1}$.

Define the following operator: $G_{F}: L^{2}(0, \tau ; U) \longrightarrow Z$ by

$$
\begin{aligned}
G_{F} u & =\int_{0}^{\tau} T(\tau-s) B u(s) d s+\int_{0}^{\tau} T(\tau-s) F(s, z(s), u(s)) d s \\
& =G u+\int_{0}^{\tau} T(\tau-s) F(s, z(s), u(s)) d s
\end{aligned}
$$

where $z(t)=z(t, u)$ is the solution of (4.14) corresponding to the control $u$. Then, the following proposition is trivial and characterizes the exact controllability of (4.1).

Proposition 2. The system (4.1) is exactly controllable on $[0, \tau]$ if, and only if,

$$
\operatorname{Rang}\left(G_{F}\right)=Z \text {. }
$$

So, in order to prove exact controllability of system (4.1) we have to verify the condition of the foregoing proposition. To this end, we need to assume that the linear system (1.3) is exactly controllable. In this case we know from corollary 1 that the steering operator $S$ defined by (4.8) is a right inverse of $G$, so if we put $G_{F}=G_{F} \circ S$, we get the following representation:

$$
G_{F} \xi=G_{F} \circ S \xi=\xi+\int_{0}^{\tau} T(\tau-s) F\left(s, z_{\xi}(s),(S \xi)(s)\right) d s
$$

where $z_{\xi}(\cdot)$ is the solution of (4.14) corresponding to 
the control $u$ define by:

$$
u(t)=(S \xi)(t)=B^{*} T^{*}(\tau-t) W^{-1} \xi, t \in[0, \tau] .
$$

Hence, if we define the operator $K: Z \longrightarrow Z$ by:

$$
K \xi=\int_{0}^{\tau} T(\tau-s) F\left(s, z_{\xi}(s),(S \xi)(s)\right) d s
$$

the operator $G_{F}$ can be written as follows

$$
G_{F} \xi=\xi+K \xi=(I+K) \xi
$$

Now, we shall prove some abstract results making assumptions on the operator $K$. After that, we will put conditions on the nonlinear term $F$ that imply condition on $K$.

Theorem 4. If the linear system (1.3) is exactly controllable on $[0, \tau]$ and the operator $K$ is globally Lipschitz with a Lipschitz constant $k<1$, then the non linear system (4.1) is exactly controllable on $[0, \tau]$ and the control steering the initial state $z_{0}$ to the final state $z_{1}$ is given by:

$$
u(t)=B^{*} T^{*}(\tau-t) W^{-1}(I+K)^{-1}\left(z_{1}-T(\tau) z_{0}\right) .
$$

Proof It follows directly from Equation (3.6) and Theorem 1 or Theorem 2 .

Theorem 5. If the system (1.3) is exactly controllable on $[0, \tau]$ and the operator $K$ is linear with $K \geq 0$, then the system (4.1) is exactly controllable on $[0, \tau]$ and the control $u(t)$ steering the initial state $z_{0}$ to the final state $z_{1}$ is given by:

$$
u(t)=B^{*} T^{*}(\tau-t) W^{-1}(I+K)^{-1}\left(z_{0}-T(\tau) z_{1}\right)
$$

Proof It follows directly from Equation (3).

The proof of the following lemma follows as in lemma 5.1 from [1].

Lemma 2. If $F$ satisfies the Lipschitz condition

$$
\begin{aligned}
& F\left(t, z_{2}, u_{2}\right)-F\left(t, z_{1}, u_{1}\right) \leq L\left\{z_{2}-z_{1}+u_{2}-u_{1}\right\} \\
& z_{2}, z_{1} \in Z \quad u_{2}, u_{1} \in U \quad t \in[0, \tau],
\end{aligned}
$$

then

$$
K z_{2}-K z_{1} \leq K(L) z_{2}-z_{1}, z_{2}, z_{1} \in Z
$$

where $K(L)=L H_{1} H_{2}$ and

$$
\begin{aligned}
& H_{1}=M[B+L] e^{M L \tau} \tau+1, H_{2}= \\
& =M^{2} B W^{-1} \tau, M=\sup _{0 \leq s \leq t \leq \tau} T(t-s) .
\end{aligned}
$$

Theorem 6. If $F$ satisfies the foregoing Lipschitz condition, the linear system (1.3) is exactly controllable on $[0, \tau]$ and

$$
L\left(M[B+L] e^{M L \tau} \tau+1\right)\left(M^{2} B W^{-1} \tau\right)<1
$$

then the non linear system (4.1) is exactly controllable on $[0, \tau]$ and the control steering the initial state $z_{0}$ to the final state $z_{1}$ is given by:

$$
u(t)=B^{*} T^{*}(\tau-t) W^{-1}(I+K)^{-1}\left(z_{1}-T(\tau) z_{0}\right) .
$$

Proof From Lemma 2 we know that $K$ is a Lipschitz function with a Lipschitz constant $k$ given by:

$$
k=L\left(M[B+L] e^{M L \tau} \tau+1\right)\left(M^{2} B W^{-1} \tau\right)
$$

and from condition (4.19) we get that $k<1$. Hence, applying Theorem 4 we complete the proof.

\section{Applications and Further Research}

In this section we consider some control systems governed by partial differential equations, integrodifferential equations and difference equations that can study using these results. Particularly, we work in details the controlled damped wave equation. Finally, we propose future investigations an open problem.

\subsection{The Controlled Semilinear Damped Wave Equation}

Consider the following control system governed by a $1 D$ semilinear damped wave equation

$$
\left\{\begin{array}{l}
w_{t t}+c w_{t}-d w_{x x}=u(t, x)+f\left(t, u(t, x), w, w_{t}\right) \\
0<x<1 \\
w(t, 0)=w(t, 1)=0 \\
t \in I R
\end{array}\right.
$$

where $d>0, c \geq 0$, the distributed control $u \in L^{2}\left(0, t_{1} ; L^{2}(0,1)\right)$ and the nonlinear term $f(t, w, v, u)$ is a function $f:\left[0, t_{1}\right] \times I R^{3} \rightarrow I R$.

Abstract Formulation of the Problem.

Now we will choose the space in which problem (5.1) will be set as an abstract first order ordinary differential equation.

Let $X=L^{2}[0,1]$ and consider the linear unbounded operator

$$
\begin{gathered}
A: D(A) \subset X \rightarrow X \text { defined by } A \varphi=-\varphi_{x x} \text {, where } \\
D(A)=\left\{\varphi \in X: \varphi, \varphi_{x} \text {, are a.c, } \varphi_{x x} \in X ; \varphi(0)=\varphi(1)=0\right\} .
\end{gathered}
$$

The operator $A$ has the following very well known properties:

1) The spectrum of $A$ consists of only eigenvalues

$$
0<\lambda_{1}<\lambda_{2}<\cdots<\lambda_{n} \rightarrow \infty
$$

each one with multiplicity one.

2) There exists a complete orthonormal set $\left\{\varphi_{n}\right\}$ of eigenvectors of $A$.

3) For all $x \in D(A)$ we have

$$
A x=\sum_{n=1}^{\infty} \lambda_{n}<x, \varphi_{n}>\varphi_{n}=\sum_{n=1}^{\infty} \lambda_{n} E_{n} x,
$$

where $\langle\cdot, \cdot\rangle$ is the inner product in $X$ and 


$$
\begin{aligned}
& E_{n} x=<x, \varphi_{n}>\varphi_{n}, \lambda_{n}=n^{2} \pi^{2} \\
& \text { and } \varphi_{n}(x)=\sqrt{2} \sin (n \pi x) .
\end{aligned}
$$

So, $\left\{E_{n}\right\}$ is a family of complete orthogonal projections in $X$ and

$$
x=\sum_{n=1}^{\infty} E_{n} x, x \in X .
$$
by:

4) - Agenerates an analytic semigroup $\left\{e^{-A t}\right\}$ given

$$
e^{-A t} x=\sum_{n=1}^{\infty} e^{-\lambda_{n} t} E_{n} x
$$

and

5) The fractional powered spaces $X^{r}$ are given by:

$$
X^{r}=D\left(A^{r}\right)=\left\{x \in X: \sum_{n=1}^{\infty}\left(\lambda_{n}\right)^{2 r} E_{n} x^{2}<\infty\right\} r \geq 0,
$$

with the norm

$$
x_{r}=A^{r} X=\left\{\sum_{n=1}^{\infty} \lambda_{n}^{2 r} E_{n} x^{2}\right\}^{1 / 2}, x \in X^{r}
$$

where

$$
A^{r} X=\sum_{n=1}^{\infty} \lambda_{n}^{r} E_{n} x
$$

Also, for $r \geq 0$ we define $Z_{r}=X^{r} \times X$, which is a Hilbert Space with norm given by:

$$
\left\|\left[\begin{array}{c}
w \\
v
\end{array}\right]\right\|_{Z_{r}}^{2}=w_{r}^{2}+v^{2} .
$$

Using the change of variables $w^{\prime}=v$, the system (5.1) can be written as a first order systems of ordinary differential equations in the Hilbert space

$$
\begin{aligned}
& Z_{1 / 2}=D\left(A^{1 / 2}\right) \times X=X^{1 / 2} \times X \text { as: } \\
& \quad z^{\prime}=A z+B u+F(t, z, u(t)), z \in Z_{1 / 2}, t \geq 0,
\end{aligned}
$$

where

$$
z=\left[\begin{array}{l}
w \\
v
\end{array}\right], B=\left[\begin{array}{c}
0 \\
I_{X}
\end{array}\right], A=\left[\begin{array}{cc}
0 & I_{X} \\
-d A & -C I_{X}
\end{array}\right],
$$

is an unbounded linear operator with domain $D(A)=D(A) \times X$ and

$$
F(t, z, u)=\left[\begin{array}{c}
0 \\
f(t, u, w, v)
\end{array}\right]
$$

and the function $\mathrm{F}$ : $\left[0, \mathrm{t}_{1}\right] \times Z_{1 / 2} \times X \rightarrow Z_{1 / 2}$. Since $X^{1 / 2}$ is continuously included in $X$ we obtain for all $z_{1}, z_{2} \in Z_{1 / 2}$ and $u_{1}, u_{2} \in X$ that

$$
\begin{aligned}
& F\left(t, z_{2}, u_{2}\right)-F\left(t, z_{1}, u_{1}\right)_{Z_{1 / 2}} \\
& \leq L\left\{z_{2}-z_{1 / 2}+u_{2}-u_{1}\right\} \quad t \in\left[0, t_{1}\right] .
\end{aligned}
$$

Throughout this section, without lose of generality, we will assume that

$$
c^{2}<4 d \lambda_{1} .
$$

The following proposition follows from [8] and [1].

Proposition 1. The operator $A$ given by (5.8), is the infinitesimal generator of strongly continuous group $\{T(t)\}_{t \in I R}$ in $Z_{1 / 2}$ given by:

$$
T(t) z=\sum_{n=1}^{\infty} e^{A_{n} t} P_{n} z, z \in Z_{1 / 2},
$$

where $\left\{P_{n}\right\}_{n \geq 0}$ is a family of complete orthogonal projections on the Hilbert space $\mathbf{Z}_{\frac{1}{2}}$ given by:

$$
P_{n}=\operatorname{diag}\left(E_{n}, E_{n}\right), n \geq 1,
$$

and

$$
A_{n}=B_{n} P_{n}, B_{n}=\left(\begin{array}{cc}
0 & 1 \\
-d \lambda_{n} & -C
\end{array}\right), n \geq 1 .
$$

This group decays exponentially to zero. In fact, we have the following estimate

$$
T(t) \leq M(c, d) e^{-\frac{c}{2} t}, t \geq 0,
$$

where

$$
\frac{M(c, d)}{2 \sqrt{2}}=\sup _{n}\left\{2\left|\frac{c \pm \sqrt{4 d \lambda_{n}-c^{2}}}{\sqrt{c^{2}-4 d \lambda_{n}}}\right|,(2+d)\left|\frac{\sqrt{\lambda_{n}}}{\sqrt{4 d \lambda_{n}-c^{2}}}\right|\right\}
$$

The proof of the following theorem follows in the same way as the one for Theorem 4.1 from [1].

Theorem 1. The system

$$
\left\{\begin{array}{l}
z^{\prime}=A z+B u z \in Z_{1 / 2}, t>0, \\
z(0)=z_{0} .
\end{array}\right.
$$

is exactly controllable on $[0, \tau]$.

Theorem 2. If the following estimate holds

$$
L\left(M(c, d)[1+L] e^{M(c, d) L \tau} \tau+1\right)\left(M(c, d)^{2} W^{-1} \tau\right)<1,
$$

then the system (5.7) is exactly controllable on $[0, \tau]$.

Proof It follows from Theorem 6 one we observe that in this case $B \leq 1$.

\subsection{Future Research}

These results can be applied to the following class of second order diffusion system in Hilbert spaces

$$
\begin{aligned}
& w^{\prime \prime}+A_{0} w=u(t)+f(t, w, u) \\
& t>0, \quad w \in W \quad u \in U
\end{aligned}
$$


Where $W, U$ are Hilbert spaces, the control $u \in L^{2}(0, \tau ; U)$, $A_{0}: D\left(A_{0}\right) \subset W \rightarrow W$ is an unbounded linear operator in $W$ with the spectral decomposition:

$$
A_{0} w=\sum_{j=1}^{\infty} \lambda_{j} \sum_{k=1}^{\gamma_{j}}<\phi_{k, j}, w>\phi_{k, j}=\sum_{j=1}^{\infty} \lambda_{j} E_{j} w,
$$

where $E_{j} w=\sum_{k=1}^{\gamma_{j}}<\phi_{k, j}, w>\phi_{k, j},\left\{\varphi_{k, j}\right\}$ is a complete orthonormal set of eigenvectors of $-A_{0}$ correspondent to the eigenvalues $\lambda_{1}<\lambda_{2}<\cdots<\lambda_{n} \rightarrow \infty$ with multiplicity $\gamma_{n}$ and $-A_{0}$ generates a strongly continuous semigroup $\{T(t)\}_{t \geq 0}$ given by:

$$
T(t) w=\sum_{j=1}^{\infty} e^{-\lambda_{j} t} E_{j} w, w \in W, t \geq 0
$$

and $f:[0, \tau] \times W \times U \longrightarrow W$ is a suitable function.

Examples of this class are the following well known systems of partial differential equations:

Example 1. The $n \mathrm{D}$ wave equation with Dirichlet boundary conditions

$$
\left\{\begin{array}{l}
\frac{\partial^{2} w}{\partial^{2} t}-\Delta w=u(t, x)+f(t, u(t, x), w) \\
t \geq 0, x \in \Omega \\
w(t, x)=0 \\
t \geq 0, x \in \partial \Omega \\
w(0, x)=\varphi_{0}(x) \\
\frac{\partial z}{\partial t}(0, x)=\psi_{0}(x) \\
x \in \Omega
\end{array}\right.
$$

where $\Omega$ is a sufficiently smooth bounded domain in $I R^{N}, u \in L^{2}\left([0, r] ; L^{2}(\Omega)\right), \varphi_{0}, \psi_{0} \in L^{2}(\Omega)$ and $f$ is a suitable function.

Example 2. The model of Vibrating Plate

$$
\left\{\begin{array}{l}
\frac{\partial^{2} w}{\partial^{2} t}+\Delta^{2} w+=u(t, x)+f(t, u(t, x), w) \\
t \geq 0, x \in \Omega \\
w=\Delta w=0 \\
t \geq 0, x \in \partial \Omega . \\
w(0, x)=\varphi_{0}(x) \\
\frac{\partial w}{\partial t}(0, x)=\psi_{0}(x) \\
x \in \Omega .
\end{array}\right.
$$

where $\Omega$ is a sufficiently smooth bounded domain in
$I R^{2}, u \in L^{2}\left([0, r] ; L^{2}(\Omega)\right), \quad \varphi_{0}, \psi_{0} \in L^{2}(\Omega)$ and $f$ is a suitable function.

Others type of problems are the following control problems:

Example 3. Interior Controllability of the 1D Wave Equation

$$
\begin{cases}y_{t t}-y_{x x}=1_{\omega} u(t, x)+f\left(t, u(t, x), w, w_{t}\right) \\ \text { in }(0, \tau) \times(0,1) \\ y(t, 0)=y(t, 1)=0, & t \in(0, \tau) \\ y(0, x)=y_{0}(x), & \text { in }[0,1]\end{cases}
$$

where $\omega$ is an open nonempty subset of $[0,1], 1_{\omega}$ denotes the characteristic function of the set $\omega$, the distributed control $u \in L^{2}\left(0, \tau ; L^{2}[0,1]\right)$ and the nonlinear term $f(t, w, v, u)$ is a function $f:\left[0, t_{1}\right] \times I R^{3} \rightarrow I R$. For the interior controllability of the linear wave equation one can see [5].

Example 4. Exact Controllability of Integrodifferential 1D Wave Equation with Delay.

$$
\left\{\begin{array}{l}
y_{t t}-y_{x x}=u(t, x)+\int_{0}^{t} p(s, y(s-r, x) d s \\
\text { in }(0, \tau) \times(0,1) \\
y(t, 0)=y(t, 1)=0 \\
t \in(0, \tau) \\
y(s, x)=y_{0}(s, x) \\
\text { in }[-r, 0] \times[0,1] \\
y_{t}(s, x)=y_{1}(s, x) \\
\text { in }[-r, 0] \times[0,1]
\end{array}\right.
$$

where the distributed control $u \in L^{2}\left(0, \tau ; L^{2}[0,1]\right)$, $y_{0}, y_{1}:[-r, 0] \times[0,1] \longrightarrow I R$ are continuous functions and the nonlinear term $p \in L^{1}([-r, \tau] \times[0,1])$.

Example 5. Exact controllability of Semilinear Difference Equations

$$
\begin{aligned}
& z(n+1)=A(n) z(n)+B(n) u(n)+ \\
& f(z(n), u(n)), n \in I N^{*}, z(0)=z_{0}
\end{aligned}
$$

where $Z, U$ are Hilbert spaces, $A \in l^{\infty}(I N, L(Z))$, $B \in l^{\infty}(I N, L(U, Z)), \quad u \in l^{2}(I N, U), \quad L(U, Z)$ denotes the space of all bounded linear operators from $U$ to $Z$ and $L(Z, Z)=L(Z)$. The nonlinear term $f: Z \times U \longrightarrow Z$ is a continuous Lipschitzian function. That is to say: For all $z_{2}, z_{1} \in Z$ and $u_{1}, u_{2} \in U$ we have that

$$
f\left(z_{2}, u_{2}\right)-f\left(z_{1}, u_{1}\right) \leq L\left\{z_{2}-z_{1}+u_{2}-u_{1}\right\} .
$$




\subsection{Open Problem}

The solution of the following problem is very important, no only to study the approximate controllability of evolution Equation (1.2), it also can be used to solve others mathematical problems.

The problem can be formulate as follows: Let $Z, W$ be Hilbert spaces, $G \in L(W, Z)$ and $H: W \longrightarrow Z$ is a suitable nonlinear function. When the following statement holds?

If $\overline{\operatorname{Rang}(G)}=Z$ and $H$ is a Lipschitz function with a Lipschitz constant $h$ small enough, then

$\overline{\operatorname{Rang}(G+H)}=Z$ and for all $z \in Z$ there is a sequence $\left\{w_{\alpha}\right\}_{\alpha>0} \subset W$ such that equation

$$
\lim _{\alpha \rightarrow 0^{+}}\left(G w_{\alpha}+H\left(w_{\alpha}\right)\right)=z \text {. }
$$

\section{References}

[1] H. Leiva, "Exact Controllability of the Suspension Bridge Model Proposed by Lazer and Mckenna," Journal of Mathematical Analysis and Applications, Vol. 309, No. 2, 2005, pp. 404-419.

[2] E. Iturriaga and H. Leiva, "A Necessary and Sufficient
Condition for the Controllability of Linear Systems in Hilbert Spaces and Applications," IMA Journal of Mathematics, Control and Information, Vol. 25, No. 3, 2008, pp. 269-280.

[3] H. Leiva, "Exact Controllability of Semilinear Evolution Equation and Applications," International Journal of Systems and communications, Vol. 1, No. 1, 2008, pp. 112.

[4] H. Leiva and J. Uzcategui, "Exact Controllability of Semilinear Difference Equation and Application,” Journal of Difference Equations and Applications, Vol. 14, No. 7, 2008, pp. 671-679.

[5] J. L. Lions, "Optimal Control of Systems Governed by Partial Differential Equations,” Die Grundlehren der mathematischen Wissenschaften in Einzeldarstellungen Band 170, 1971.

[6] R. F. Curtain and A. J. Pritchard, "Infinite Dimensional Linear Systems," Lecture Notes in Control and Information Sciences, Vol. 8, Springer Verlag, Berlin, 1978.

[7] R. F. Curtain and H. J. Zwart, “An Introduction to Infinite Dimensional Linear Systems Theory," Tex in Applied Mathematics, Vol. 21. Springer Verlag, New York, 1995.

[8] H. Leiva, "A Lemma on $\mathrm{C}_{0}$-Semigroups and Applications PDEs Systems,” Quaestions Mathematicae, Vol. 26, No. 3, 2003, pp. 247-265. 